\title{
Multi-Lepton and Isolated Lepton Events at HERA
}

\author{
David M. South ${ }^{* i}$ \\ Technische Universität Dortmund \\ Experimentelle Physik $V$ \\ 44221 Dortmund, Germany \\ E-mail: david.south@desy.de
}

\begin{abstract}
Measurements of the production of events containing isolated high energy leptons (electrons and muons) produced in $e p$ collisions have been performed with the H1 and ZEUS detectors at HERA, using data collected in the period $1994-2007$. Topologies with more than one charged lepton or with a charged lepton in coincidence with missing transverse momentum are analysed. The $\mathrm{H} 1$ and ZEUS data, corresponding to an integrated luminosity of about $0.5 \mathrm{fb}^{-1}$ per experiment, are combined in a common phase space. The observed event yields are compared to the predictions from the Standard Model. In general a good agreement is found, where the expectation is dominated by photon-photon collisions for the multi-lepton topologies and by single $W$ production in the case of events with an isolated electron or muon and missing transverse momentum. Events with large transverse momentum are observed. Total and differential cross sections of these processes are measured.
\end{abstract}

European Physical Society Europhysics Conference on High Energy Physics, EPS-HEP 2009, July 16 - 222009

Krakow, Poland

\footnotetext{
*Speaker.

${ }^{\dagger}$ on behalf of the H1 and ZEUS Collaborations
} 


\section{Introduction}

The electron-proton collisions at HERA provide a unique opportunity to look for physics beyond the Standard Model. Events with one or more isolated leptons in the final state, as well as in combination with missing transverse momentum, maybe a signature for rare processes. The good lepton identification and hadronic final state reconstruction of the $\mathrm{H} 1$ and ZEUS experiments means that such topologies provide a clean signal. The Standard Model (SM) expectation for such events at HERA is low, so the analysis benefits from the combination of the H1 and ZEUS data, resulting in a total integrated luminosity of about $1 \mathrm{fb}^{-1}$.

\section{Multi-Lepton Events}

The production of multi-lepton final states in $e p$ collisions proceeds in the SM mainly via photon-photon interactions. Measurements of multi-lepton production at HERA have been performed by the H1 [1] and ZEUS [2] collaborations. A combined analysis of the H1 and ZEUS data is performed in a common phase space, using the full data samples available to both experiments [3].

Electrons are identified in the polar-angle range $5^{\circ}<\theta_{e}<175^{\circ}$ with $E_{e}>10 \mathrm{GeV}$ in the range $5^{\circ}<\theta_{e}<150^{\circ}$ and $E_{e}>5 \mathrm{GeV}$ in the backward region $150^{\circ}<\theta_{e}<175^{\circ}$. Muon candidates are identified in the range $20^{\circ}<\theta_{\mu}<160^{\circ}$ with $P_{T}^{\mu}>2 \mathrm{GeV}$. All lepton candidates are required to be isolated with respect to each other by a minimum distance of at least 0.5 units in the $\eta-\phi$ plane. At least two central $\left(20^{\circ}<\theta<150^{\circ}\right)$ lepton candidates are required, one of which must have $P_{T}^{\ell}>10 \mathrm{GeV}$ and the other $P_{T}^{\ell}>5 \mathrm{GeV}$. Additional leptons identified in the detector according to the criteria defined above may be present in the event. According to the number and the flavour of the lepton candidates, the events are classified into mutually exclusive topologies. A full description of the common phase space event selection is presented in [3].

A good overall agreement is observed with the SM in all event topologies, where the SM prediction in the $e \mu \mu, \mu \mu$ and $e \mu$ topologies is dominated by muon pair production while the eee and ee topologies contain mainly events from electron pair production. For events where $\sum P_{T}^{\ell}>100 \mathrm{GeV}$, seven events are observed in the data, compared to $3.13 \pm 0.26$ expected from the SM, as shown in table 1. All seven events were recorded in the $e^{+} p$ data, for which the SM expectation is $1.94 \pm 0.17$. The lepton-pair production cross section is measured in a phase space dominated by gamma-gamma interactions as $0.66 \pm 0.03$ (stat.) \pm 0.03 (sys.) pb, in agreement with the SM prediction of $0.69 \pm 0.02 \mathrm{pb}$. The cross section is also measured as a function of $P_{T}^{\ell_{1}}$ and the invariant mass of the lepton pair $M_{\ell \ell}$, as shown in figure 1 .

\begin{tabular}{ccccc} 
Data sample & Data & SM & Lepton Pair Production & Background \\
\hline $\mathrm{e}^{+} \mathrm{p}\left(0.56 \mathrm{fb}^{-1}\right)$ & 7 & $1.94 \pm 0.17$ & $1.52 \pm 0.14$ & $0.42 \pm 0.07$ \\
$\mathrm{e}^{-} \mathrm{p}\left(0.38 \mathrm{fb}^{-1}\right)$ & 0 & $1.19 \pm 0.12$ & $0.90 \pm 0.10$ & $0.29 \pm 0.05$ \\
All $\left(0.94 \mathrm{fb}^{-1}\right)$ & 7 & $3.13 \pm 0.26$ & $2.42 \pm 0.21$ & $0.71 \pm 0.10$ \\
\hline
\end{tabular}

Table 1: Observed and predicted multi-lepton event yields for $\sum P_{T}>100 \mathrm{GeV}$ in $0.94 \mathrm{pb}^{-1}$ of HERA data. Di-lepton and tri-lepton events are combined. The uncertainties on the predictions include model uncertainties and experimental systematic uncertainties added in quadrature. 


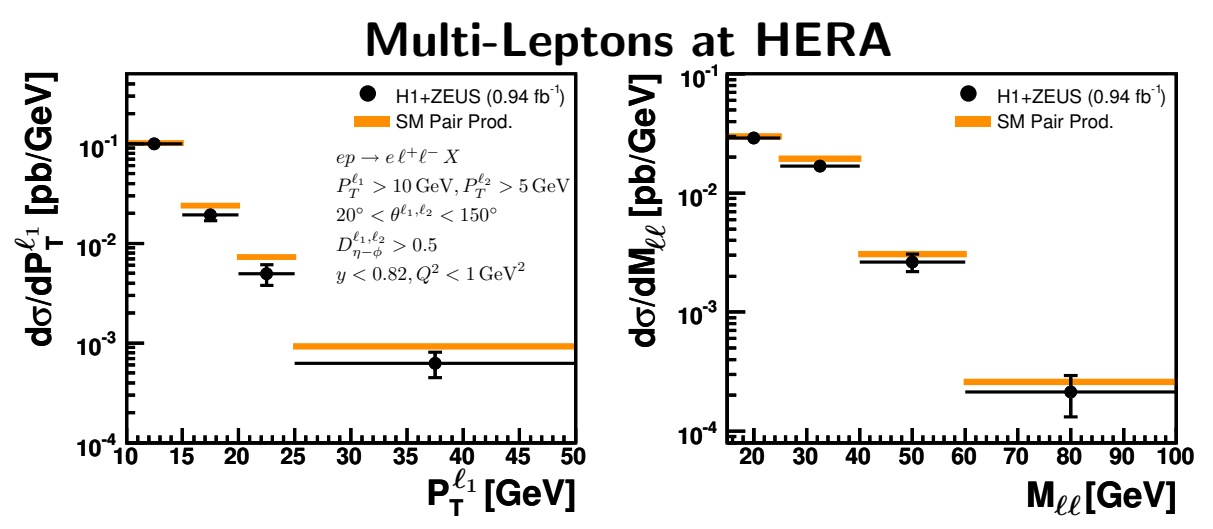

Figure 1: The cross section for lepton-pair photoproduction in a restricted phase space as a function of the leading lepton transverse momentum $P_{T}^{\ell_{1}}$ (a) and the invariant mass of the lepton pair $M_{\ell \ell}$ (b). The total error bar is shown, representing the statistical and systematic uncertainties added in quadrature. The bands represent the uncertainty in the SM prediction.

\section{Events with Isolated Leptons and Missing Transverse Momentum}

In the SM, events containing a high $P_{T}$ isolated electron or muon and large missing transverse momentum, $P_{T}^{\text {miss }}$, arise in $e p$ collisions from the production of single $W$ bosons with subsequent decay to leptons. Events of this topology have been observed at HERA [4, 5]. A combined analysis of the $\mathrm{H} 1$ and ZEUS data has recently been performed in a common phase space, which makes use of the full data samples available to both experiments [6].

The event selection is based on those used by H1 [4] and ZEUS [5]. Isolated lepton candidates are required to have $P_{T}^{\ell}>10 \mathrm{GeV}$, to be in the central region of the detector $15^{\circ}<\theta_{\ell}<120^{\circ}$ and to be isolated from tracks and identified jets in the event. The event must also exhibit significant missing transverse momentum, $P_{T}^{\text {miss }}>12 \mathrm{GeV}$. Further topological and kinematic cuts are then applied to reject the remaining SM background. A full description of the common phase space event selection is presented in [6].

The results of the analysis are shown in table 2. In general, a good agreement is observed between the data and the SM predictions, where the signal component, dominated by single $W$ production, forms the main part of the expectation. The lepton-neutrino transverse mass distribution is shown in figure 2 (left). For $P_{T}^{X}>25 \mathrm{GeV}, 29$ events are observed in the data, compared to a SM prediction of $24.0 \pm 3.2$ for the complete HERA $e^{ \pm} p$ data. In the $e^{+} p$ data alone, where a small excess of data is seen in $\mathrm{H} 1$ analysis [4], 23 events are observed in the data, compared to a SM prediction of $14.0 \pm 1.9$. The single $W$ cross section is measured as $1.06 \pm 0.16$ (stat.) \pm 0.07 (sys.) pb, in agreement with the SM prediction of $1.26 \pm 0.19 \mathrm{pb}$. The cross section is also measured as a function of $P_{T}^{X}$, as shown in figure 2 (right).

\section{Summary}

Analyses of events with multi-leptons and isolated leptons with $P_{T}^{\text {miss }}$ have been recently published individually by $\mathrm{H} 1$ and ZEUS. Combined H1 and ZEUS analyses are also now performed in a common phase space, to take advantage of the complete HERA high energy data. In general a good agreement is observed with the SM predictions. The cross sections of multi-lepton and single 


\begin{tabular}{ccccrr} 
Channel & & Data & SM & Signal $(\mathrm{W})$ & Background \\
\hline Electron & Total & 61 & $69.2 \pm 8.2$ & $48.3 \pm 7.4$ & $20.9 \pm 3.2$ \\
& $P_{T}^{X}>25 \mathrm{GeV}$ & 16 & $13.0 \pm 1.7$ & $10.0 \pm 1.6$ & $3.1 \pm 0.7$ \\
\hline Muon & Total & 20 & $18.6 \pm 2.7$ & $16.4 \pm 2.6$ & $2.2 \pm 0.5$ \\
& $P_{T}^{X}>25 \mathrm{GeV}$ & 13 & $11.0 \pm 1.6$ & $9.8 \pm 1.6$ & $1.2 \pm 0.3$ \\
\hline Combined & Total & 81 & $87.8 \pm 11.0$ & $64.7 \pm 9.9$ & $23.1 \pm 3.3$ \\
& $P_{T}^{X}>25 \mathrm{GeV}$ & 29 & $24.0 \pm 3.2$ & $19.7 \pm 3.1$ & $4.3 \pm 0.8$ \\
\hline
\end{tabular}

Table 2: Observed and predicted number of events with an isolated electron or muon and missing transverse momentum in $0.98 \mathrm{fb}^{-1}$ of HERA data. The results are shown for the full selected sample and for the subsample at large hadronic transverse momentum $P_{T}^{X}>25 \mathrm{GeV}$. The quoted errors contain statistical and systematic uncertainties added in quadrature.
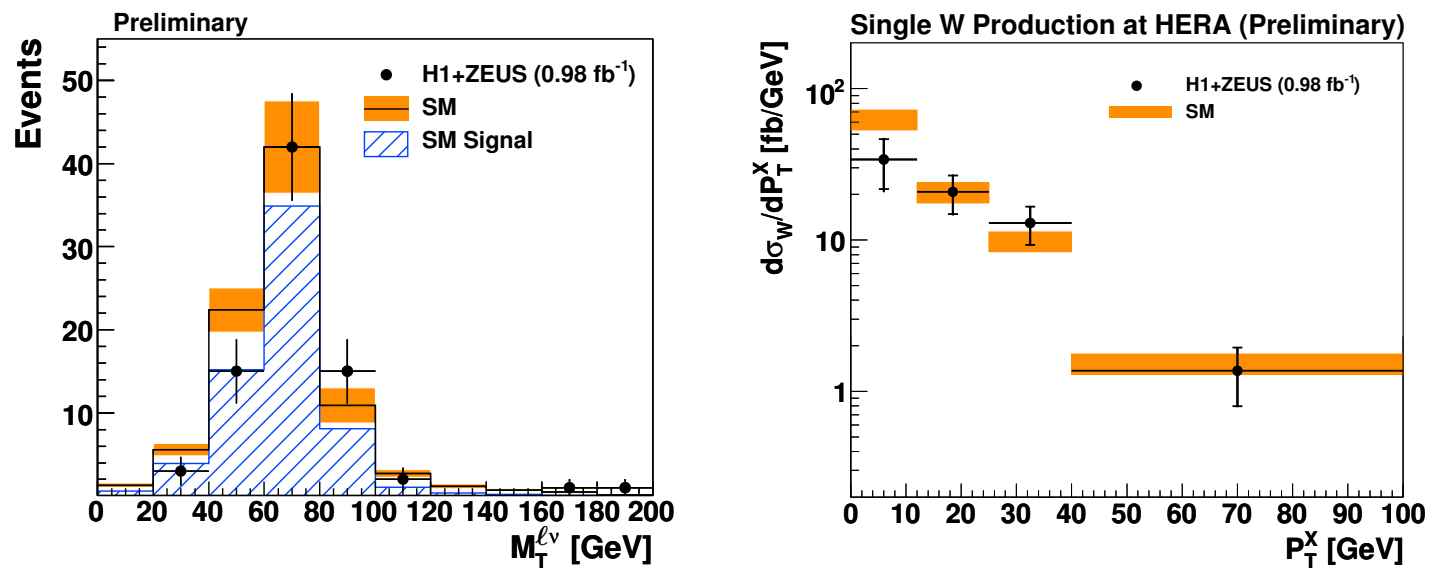

Figure 2: Left: The lepton-neutrino transverse mass $M_{T}^{\ell v}$ of events with an isolated electron or muon and missing transverse momentum. The data (points) are compared to the SM expectation (open histogram). The signal component of the SM expectation, dominated by single $W$ production, is shown as the striped histogram. The total uncertainty on the SM expectation is shown as the shaded band. Right: The single $W$ production cross section as a function of the hadronic transverse momentum, $P_{T}^{X}$. The inner error bar represents the statistical error and the outer error bar indicates the statistical and systematic uncertainties added in quadrature. The shaded band represents the uncertainty on the SM prediction.

$W$ production are measured with a greater statistical precision. A few events are observed by both $\mathrm{H} 1$ and ZEUS in the $e^{+} p$ data at high $P_{T}$ and high mass, a region where the SM expectation is low.

\section{References}

[1] F. D. Aaron et al. [H1 Collaboration], Phys. Lett. B668 (2008) 268, [arXiv:0806.3987].

[2] S. Chekanov et al. [ZEUS Collaboration], accepted by Phys. Lett. B, arXiv:0906.1504.

[3] F. D. Aaron et al. [H1 and ZEUS Collaborations], accepted by JHEP, arXiv:0907.3627.

[4] F. D. Aaron et al. [H1 Collaboration], accepted by Eur. Phys. J. C, arXiv:0901.0488.

[5] S. Chekanov et al. [ZEUS Collaboration], Phys. Lett. B672 (2009) 106 [arXiv:0807.0589].

[6] H1 and ZEUS Collaborations, H1prelim-09-061/ZEUS-prel-09-014. 